\title{
Concurrent training effects on heart rate variability, blood pressure and fitness of middle-aged men and women
}

http://dx.doi.org/10.11606/1807-5509202000040543

\author{
Melissa Antunes* \\ Valéria Bonganha* \\ Giovana Verginia de Souza ${ }^{* *}$ \\ Arthur Fernandes Gaspari ** \\ Cleiton A. Libardi ${ }^{* * *}$ \\ Mara Patrícia T. Chacon-Mikahil ${ }^{* *}$
}

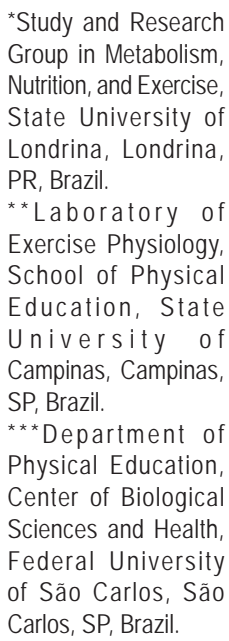

*Study and Research Group in Metabolism, Nutrition, and Exercise, State University of Londrina, Londrina, PR, Brazil.

** Laboratory of Exercise Physiology, School of Physical Education, State

University of Campinas, Campinas, SP, Brazil.

***Department of Physical Education, Center of Biological Sciences and Health, Federal University of São Carlos, São Carlos, SP, Brazil.

The concurrent training led to beneficial effects on aerobic fitness and muscle strength. However, its effects on blood pressure (BP) and autonomic control marks are little studied. This study aimed to evaluate the concurrent training effects on $\mathrm{BP}$, autonomic control, aerobic fitness and muscle strength of middle-aged men and women. Thirty-two volunteers $(51.4 \pm 4.2$ years, systolic BP $115 \pm 12 \mathrm{mmHg}$ and diastolic BP 78 $\pm 8 \mathrm{mmHg}$ ) were divided into 4 groups of 8 volunteers: male concurrent training (MCT), female concurrent training (FCT), male sedentary control (MSC) and female sedentary control (FSC) groups. A minimum absence of menstruation for 12-months was required. Concurrent training, six resistance exercise for whole body followed by 30 min of jogging and/or running at 55\%-85\% of $\mathrm{VO}_{2 \text { peak' }}$ was performed three times a week. The main assessments were rest BP and cardiovascular autonomic markers evaluated through heart rate variability (iRR, LF, HF, LF/HF, RMSSD, pNN50, SD1 and SD2), aerobic fitness measured by cardiorespiratory test $\left(\mathrm{VO}_{2 \text { peak }}\right)$ and muscle strength by one repetition maximum (1-RM - arm curl, bench press and leg press). The concurrent training did not change any cardiovascular variables. Regarding fitness the MCT and FCT groups demonstrated significant improvement in $\mathrm{VO}_{2 \text { peak }}(13.12 \%$ e $8.51 \%$, respectively). Muscle strength improved significantly in the MCT group in all three exercises (arm curl: 26.53\%; bench press: 25.04\%; leg press: 65.37\%), while FCT in just two exercises (arm curl: 12.79\%; bench press: 17.25\%). Although concurrent training appears to be a good alternative for inducing improvements in various physical fitness variables in male, its can induce concurrence in leg strength of female. Concurrent training is not an effective strategy to improve BP and autonomic nervous system.

KEYwords: Physical training; Gender; Autonomic nervous system; Middle age.

\section{Introduction}

Aging related reduction in cardiovascular health is affect by lifestyles and sex ${ }^{1}$. The relationship between increase physical activity and aerobic training and fitness with risk reduction of chronic diseases and mortality is undeniable ${ }^{2,3}$, and likewise a growing body of evidence has suggested the same for resistance training ${ }^{4}$. In this regarding the combining resistance with aerobic training (known as concurrent training) has been recommended due to its beneficial effects on aerobic fitness and body strength ${ }^{5-7}$. However, its effects on cardiovascular health marks, as blood pressure (BP) and arterial stiffness are reduced when compared to aerobic training alone $e^{8,9}$. Although the autonomic nervous system coordinate several factor of the cardiovascular system $^{10,11}$, the effects of concurrent training protocols on autonomic control is little studied ${ }^{12}$.

Cardiovascular autonomic control decline is highly associated with increasing age $\mathrm{e}^{13,14}$ and is also 
related to level of fitness ${ }^{14-18}$. Although a decline in autonomic control occurs in both genders, it can take place earlier in men due to the cardioprotective effect of estrogen ${ }^{19}$. Some studies indicate greater parasympathetic modulation and lower sympathetic modulation in women compared to men ${ }^{20}$. However another study ${ }^{21}$, have showed no significant differences in autonomic modulation between genders. There is evidence that these cardiovascular differences between men and women until middle age tend to disappear over the years ${ }^{20,22}$. To the best of our knowledge, just one study have shown that concurrent training may improve heart rate variability (HRV) and HR at rest, markers of autonomic control ${ }^{23}$. Interestingly, this study investigated only men who are known to have more cardiovascular benefits, as reduction in BP and improvements in autonomic control, arising from exercises training ${ }^{8,23}$. These apparently sex difference in

\section{Methods}

\section{Experimental design}

The investigation was performed over a period of 16 weeks, this protocol was divided into two phases, each lasting eight consecutive weeks. The cardiorespiratory assessment, muscular strength test and rest cardiovascular evaluation were performed before and after the 16 weeks of experimental protocol keeping a minimum of 48 hours interval between teste and last training session. The training was performed three times a week and progressively supervised and sedentary group did not perform any type of physical exercise during this period.

\section{Ethical aspects}

After meetings with volunteer groups, those with the necessary prerequisites completed an initial interview and signed the Informed Consent approved by the Ethics Committee of the local University (CEP no. 250 and 251/2003, with addendums in 2007).

\section{Participants}

No sample size calculation was performed in this study. Thirty-two volunteers aged 40-60 years were included in the study, classified as non-active according to the Baecke questionnaire ${ }^{28}$, and IPAQ ${ }^{29}$. The exclusion criteria adopted were: any complication that
HRV response to concurrent training is reinforce for a second study conducted again by the Karavirta ${ }^{24}$ group showing that 40 to 65 years old women did not improve autonomic control after similar concurrent training.

Thus, the effects of concurrent training in autonomic control markers of female, as well as the sex difference in training induce adaptation need to be investigated. The present study analyzes cardiac autonomic adaptations in response to 16 weeks of concurrent training and compares the results of male and female. Based on the above considerations, we hypothesizes that the training groups would show similar cardiorespiratory and neuromuscular adaptations, and further improvements in HRV parameters in both genders, with no differences between them, since the middle-age female volunteers of this study were postmenopausal women who have the same levels of autonomic control imbalance of the middle-age male volunteers ${ }^{19,25-27}$.

could be classified as a risk factor for proposed program, detected in the clinical evaluation, biochemistry and/or during exercise; use of any medications that interfere in physiological responses to testing; non-availability of the volunteer to participate in the experimental procedures and/or training sessions.

For the female group there were additional criteria, such as a minimum absence of menstruation for 12-months, being postmenopausal and not using any type of hormone replacement therapy. Only volunteers who participated in at least $85 \%$ of the training sessions and were not absent for more than two consecutive training sessions were included in the final sample. The groups were separated by gender into the following groups: male concurrent training (MCT), female concurrent training (FCT), male sedentary control (MSC) and female sedentary control (FSC) groups. TABLE 1 presents the general characteristics of the groups.

The volunteers who took part in the sedentary group were instructed not to practice any type of systematic physical activity during the period of the study. FIGURE 1 shows the flow of study participants.

\section{Experimental protocols}

Prior to the evaluation protocols, familiarization with the testing equipment was performed. Both the initial evaluations and re-evaluations were applied in 
the same period of the day to avoid any influence of circadian variations. All evaluations were performed at an ambient temperature of $22^{\circ} \mathrm{C}$.

Clinical exercise testing was performed initially in order to diagnose and rule out the possibility of the occurrence of cardiac events during the later maximal exercise test and the proposed physical training. A minimum of 48 hours after the clinical examination, an evaluation of total body mass (mechanical scale, Filizola, Brazil) and height (wooden stadiometer) were performed according to the procedures described by Gordon, Chumlea and Roche ${ }^{30}$.

\section{Blood pressure assessment}

Blood pressure assessments were done after approximately 10 minutes of rest using a mercury sphygmomanometer and stethoscope. The participants were positioned supine and the same professional made the measurements. All measurements were taken in duplicate and the mean of two assessments was used. The formula for mean blood pressure (MBP) assessment was:

$$
\mathrm{MBP}=\mathrm{DBP}+[0.333 \times(\mathrm{SBP}-\mathrm{DBP})],
$$

where $\mathrm{DBP}$ is diastolic blood pressure and SBP is systolic blood pressure.

\section{Cardiovascular assessment at rest}

A minimum of 72 hours after the assessment of muscle strength, evaluation of HR was performed for the analysis of heart rate variability at rest, using a heart rate monitor (S810i - Polar - Finland) to obtain the beat-to-beat records corresponding to the intervals between consecutive $R$ waves of the electrocardiogram $(\mathrm{iRR})^{31,32}$. The volunteers were requested not to talk and to stay awake during the protocol. After five minutes of rest in the supine position, the recording was initiated and continued for a total of 30 minutes while the participant breathed spontaneously. Both the blood pressure and resting HR were measured immediately prior to the iRR collection to ensure the standard conditions of supine rest. Analysis of HRV in the time and frequency domains was performed through tachograms of iRR.

The first 10 minutes were discarded from the analysis and the most stable 256 consecutive points from the central region of the time series signal were selected by visual selection ${ }^{33}$. HRV Analysis ${ }^{\circ}$ software, version 2.0 (Finland, 2008), was used to analyze the steady state and obtain average values of $i R R$. The variables pNN50 (percentage of adjacent RR intervals with 50-millisecond difference), RMSSD (square root of the average of the squared differences between adjacent normal RR intervals) and the Poincaré plot, obtained from the SD1 and SD2 $2^{33}$ in time domain and the LF $(\mathrm{Hz})$ - low-frequency component HF $(\mathrm{Hz})$ - high-frequency component, and LF / HF (ratio low components and high frequency) values in the frequency domain.

\section{Cardiorespiratory Assessment}

The cardiorespiratory test was conducted following the descriptions used in LIBARDI et al. ${ }^{34}$. Cardiorespiratory evaluation was then performed via a test protocol on a treadmill (Quinton TM55. Bothell, Washington, USA), with an initial speed of $4 \mathrm{~km} / \mathrm{h}$ for two minutes, followed by increases of $0.3 \mathrm{~km} / \mathrm{h}$ every 30s and a constant slope of $1 \%{ }^{34}$, until physical exhaustion. Recovery was observed for a period of 4 minutes; the first minute at $5 \mathrm{~km} / \mathrm{h}$, reducing $1 \mathrm{~km} / \mathrm{h}$ every minute. Throughout the stress test, gas exchange data were collected continuously, breath to breath, by means of a metabolic gas analysis system (CPX Medical Graphics, St. Paul, Minnesota, USA). The peak oxygen consumption $\left(\mathrm{VO}_{2 \text { peak }}\right)$ was defined as the average over a period of 30 s during the final stage of the incremental test, since none of the volunteers presented the criteria used to characterize maximal oxygen consumption35. This review was carried out in three stages: before training, after eight weeks of training, only to adjust the intensity of the training program, and at the end of the 16 weeks of physical training.

\section{Muscle strength test}

A minimum of 48 hours after the cardiorespiratory evaluation, muscle strength was determined through the one repetition maximum (1-RM) test on three exercises. The order of execution of the exercises was: Elbow extension in the supine position on a horizontal bench (bench press), knee flexion and extension on a horizontal chair (leg press) and elbow flexion (arm curl), with a five minute interval between each set of exercises ${ }^{36}$. Prior to the start of the test protocol a familiarization protocol was performed in an attempt to reduce the effects of learning and establish reproducibility in the three exercises. All exercises were preceded by a warm-up series of 10 repetitions with approximately $50 \%$ of the load estimated by an experienced evaluator for the first attempt at the 
1-RM test. Testing began three minutes after the warm-up, during which the volunteers performed a single repetition with the expected load for 1-RM. Whether this was completed or not, a second attempt was given after an interval of three to five minutes with a greater or lesser load $(\mathrm{kg})$ than that previously employed. A third and final attempt was made if the single maximum repetition load had not yet been determined. To determine the results of the1RM tests at baseline, we used the value of the highest load obtained after the test-retest.

\section{Concurrent training protocol}

The concurrent training protocol was composed of aerobic and resistance training performed in the same session. The training program consisted of three weekly sessions on alternate days (Monday, Wednesday and Friday), with an approximate duration of 60 minutes per session, for a period 16 consecutive weeks. The physical training protocol was guided and accompanied by Physical Education professionals and academics. This protocol was divided into two phases, each lasting for eight consecutive weeks. During the first eight weeks of training, the participants initially performed resistance training that consisted of six exercises. They perfonned three exercises for the lower body (leg press, leg extension, and leg curl) and three exercises for the upper body (bench press, lateral pulldown, and arm curl) with three sets of 10 repetitions and intervals of one minute; the session lasted approximately 30 minutes 37 , and the order of the exercises altered by segment. Next the participants performed walking and/or continuous running for 30 minutes on an outdoor athletic track, with intensity variation during the training session as follows: five minutes below the ventilatory threshold (VT), 10 minutes at VT, 10 minutes above VT but below respiratory compensation point (RCP), five minutes below $\mathrm{VT}^{38}$, totaling over 30 minutes of training. In the subsequent eight weeks, the resistance training session was held with the same series of exercises as in the previous weeks, with eight repetitions and intervals of 90 seconds 37 , also lasting about 30 minutes, however, the order of the exercises was according to joint. For aerobic training there was an adjustment in training zone intensity and length of time in each, five minutes below the VT, 10 minutes above VT but below RCP, 10 minutes at RCP and five minutes below VT, resulting in an additional 30 minutes of training. It is noteworthy that the total duration of the concurrent training session was about 60 minutes.

After the first eight weeks, the volunteers underwent cardiopulmonary reevaluation to adjust the intensity for the next step. The aerobic intensity of training related to the VT and RCP was monitored by means of test speed on the treadmill, since it was performed with a gradient of $1 \%$ in an attempt to reproduce the training conditions on the running track. Regarding the resistance training, load adjustment was performed weekly. Adjustments were made in the final set of each exercise in the last week of the training session. Individuals were encouraged to perform the maximum number of repetitions and load was reset based on performance, using the following calculation: for each repetition exceeding the prescribed number of repetitions, the load was increased by $1 \mathrm{~kg}$ for lower limb exercises and $0.5 \mathrm{~kg}$ for upper limb and trunk exercises. Thus, the loads used for training were consistent with the stipulated maximum repetitions for each exercise by following the principle of load progression ${ }^{34}$.

\section{Statistical analysis}

Data normality was verified by the Shapiro-Wilk test. Non normal data were transformed by $\operatorname{Ln}(\mathrm{x})$ (pNN50) and $\log (\mathrm{X})$ (RMSSD, LFHF, SD1 and SD2) and then normality was confirmed. A group $X$ sex $X$ time repeated measures ANOVA was used to compare all study variables. When a significant $F$ value was found, Bonferroni post hoc was performed to localize differences. To verify training effects on cardiovascular variables independent of sex a group (training N.16 and control N.16) X time (pre- and post-16 weeks) repeated measures ANOVA was used. The software package used for all analyses was STATISTICA 6.0 (StatSoft, Inc., Tulsa, OK, USA). Data are presented as means and standard deviations of non-transformed values and the level of statistical significance was established at $\mathrm{p} \leq 0,05$. 
TABLE 1 - Mean \pm standard deviation of the general characteristics of sample in moments before and after 16 weeks of training.

\begin{tabular}{lcccc}
\hline & MSC & FSC & MCT & FCT \\
\hline $\mathrm{N}$ & 8 & 8 & 8 & 8 \\
Age (years) & $51.7 \pm 4.7$ & $52.6 \pm 4.2$ & $48.6 \pm 4.3$ & $52.5 \pm 4.1$ \\
Height $(\mathrm{cm})$ & $172.2 \pm 7.2$ & $158.5 \pm 55.7$ & $170.4 \pm 7.3$ & $160.2 \pm 4.1$ \\
Total Body Mass $(\mathrm{kg})$ & $69.5 \pm 9.5$ & $63.2 \pm 6.0$ & $85.5 \pm 9.2$ & $62.3 \pm 6.7$ \\
BMI $\left(\mathrm{kg} / \mathrm{m}^{2}\right)$ & $23.3 \pm 2.6$ & $25.1 \pm 2.2$ & $29.5 \pm 3.4$ & $24.2 \pm 2.3$ \\
& & & & \\
\hline
\end{tabular}

FIGURE 1- Flow of study participants.

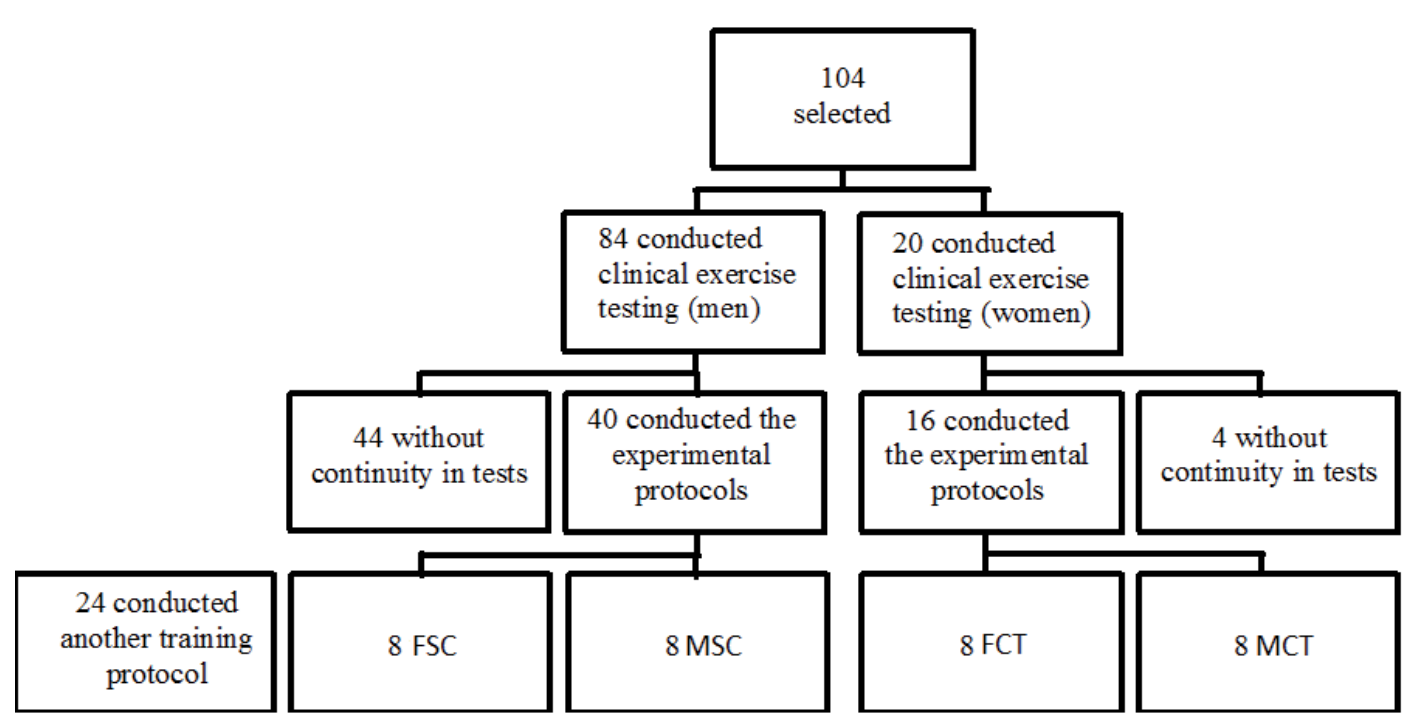




\section{Results}

The results obtained at before and after 16 weeks of training are presented as pre and post for all groups.

TABLE 2 shows heart rate (HR), systolic blood pressure (SBP), diastolic blood pressure (DBP) and mean arterial pressure (MAP) pre- and post-16 weeks for all groups. ANOVA did not show group $X$ sex $X$ time interaction (all $\mathrm{p}>0.05$ ) for any of these variable. In the same way, two way ANOVA (group $X$ time) did not show any interaction (all $\mathrm{p}>0.05$ ) for HR (training: pre- $65.6 \pm 7.7 \mathrm{bpm}$ and post -70.6 $\pm 7.2 \mathrm{bpm}$; control: pre- $70.6 \pm 7.2 \mathrm{bpm}$ and post$69.1 \pm 10.3 \mathrm{bpm}$ ), SBP (training: pre- $118.0 \pm 11.5$ $\mathrm{mmHg}$ and post- $117.1 \pm 13.2 \mathrm{mmHg}$; control: pre- $112.1 \pm 11.3 \mathrm{mmHg}$ and post $-116.9 \pm 11.2$ $\mathrm{mmHg}$ ), DBP (training: pre- $79.3 \pm 6.6 \mathrm{mmHg}$ and post- $80.1 \pm 8.8 \mathrm{mmHg}$; control: pre- $77.6 \pm$ $8.7 \mathrm{mmHg}$ and post $-79.8 \pm 8.1 \mathrm{mmHg}$ ) and MAP (training: pre- $92.2 \pm 7.6 \mathrm{mmHg}$ and post- $92.5 \pm$ $9 \mathrm{mmHg}$; control: pre- $89.1 \pm 9 \mathrm{mmHg}$ and post$92.1 \pm 8.7 \mathrm{mmHg})$.

TABLE 2- Mean \pm standard deviation of the cardiovascular variables (HR, SBP and DBP) pre- and post-16 weeks of training or sedentary control by gender.

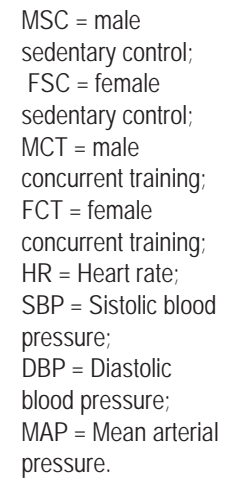

\begin{tabular}{|c|c|c|c|c|c|}
\hline & & MSC & FSC & MCT & FCT \\
\hline \multirow{2}{*}{ HR (bpm) } & pre & $71.63 \pm 8.81$ & $69.63 \pm 5.44$ & $63.13 \pm 5.99$ & $68.13 \pm 8.75$ \\
\hline & post & $68.50 \pm 10.53$ & $69.75 \pm 10.81$ & $64.50 \pm 11.77$ & $71.50 \pm 7.25$ \\
\hline \multirow{2}{*}{$\begin{array}{l}\text { SBP } \\
(\mathrm{mmHg})\end{array}$} & pre & $117.00 \pm 14.02$ & $107.25 \pm 4.52$ & $123.00 \pm 12.51$ & $113.00 \pm 8.21$ \\
\hline & post & $121.25 \pm 13.17$ & $112.50 \pm 7.07$ & $124.50 \pm 12.55$ & $109.75 \pm 9.58$ \\
\hline \multirow{2}{*}{$\begin{array}{l}\text { DBP } \\
(\mathrm{mmHg})\end{array}$} & pre & $80.75 \pm 10.69$ & $74.50 \pm 4.98$ & $79.88 \pm 6.77$ & $78.75 \pm 6.92$ \\
\hline & post & $81.50 \pm 10.18$ & $78.00 \pm 5.45$ & $84.00 \pm 4.78$ & $76.25 \pm 10.44$ \\
\hline \multirow{2}{*}{$\begin{array}{l}\text { MAP } \\
(\mathrm{mmHg})\end{array}$} & pre & $92.83 \pm 11.04$ & $85.41 \pm 4.42$ & $94.25 \pm 8.20$ & $90.16 \pm 6.87$ \\
\hline & post & $94.75 \pm 10.86$ & $89.50 \pm 5.42$ & $97.50 \pm 5.61$ & $87.41 \pm 9.08$ \\
\hline
\end{tabular}


TABLE 3 shows the hear rate variability (HRV) variables obtained pre- and post- 16 weeks of training or sedentary control by gender. It was show that none of the components of HRV presented significant group $X$ sex $X$ time interaction in ANOVA analyzes (all p > 0.05). ANOVA did not show group $X$ sex $X$ time interaction (all $p>0.05$ ) for any of these variable. In the same way, two way ANOVA (group X time) did not show any interaction (all $\mathrm{p}>0.05$ ) for iRR (training: pre- 894.6 $\pm 141.0 \mathrm{~ms}$ and post- $913.1 \pm 133.1 \mathrm{~ms}$; control: pre- $877.4 \pm 144.0 \mathrm{~ms}$ and post- $887.5 \pm 123.0 \mathrm{~ms}$ ), RMSSD (training: pre- $42.0 \pm 25.8 \mathrm{~ms}$ and post -55.8 $\pm 31.0 \mathrm{~ms}$; control: pre- $79.3 \pm 117.5 \mathrm{~ms}$ and post -45.0 $\pm 27.0 \mathrm{~ms}$ ), pNN50 (training: pre- $11.2 \pm 16.9 \%$ and post- $12.3 \pm 13.2 \%$; control: pre- $10.6 \pm 17.4 \%$ and post- $6.8 \pm 9.3 \%$ ), LF (training: pre- $61.1 \pm 14.2$ nu- $55.0 \pm 11.5 \mathrm{nu}$; control: pre- $58.9 \pm 19.0 \mathrm{nu}$ and post- $60.1 \pm 15.3 \mathrm{nu}$ ), HF (training: pre- $38.9 \pm 14.2$ nu and post $-45.5 \pm 10.6 \mathrm{nu}$; control: pre- $40.9 \pm 19.0$ nu and post- $39.8 \pm 15.3 \mathrm{nu}$ ), LFHF (training: pre- 2.2 \pm 1.3 and post $-1.7 \pm 1.4$; control: pre- $2.3 \pm 2.3$ and post- $2.1 \pm 1.4$ ), SD1 (training: pre- $30.2 \pm 18.5 \mathrm{~ms}$ and post- $40.5 \pm 22.5 \mathrm{~ms}$; control: pre- $59.4 \pm 91.7$ and post- $32.3 \pm 19.4 \mathrm{~ms}$ ), SD2 (training: pre- $68.5 \pm$ $32.2 \mathrm{~ms}$ and post- $70.4 \pm 29.6 \mathrm{~ms}$; control: pre- 90.2 \pm 92.5 and post $-65.6 \pm 21.4 \mathrm{~ms}$ ).

TABLE 3- Mean values \pm standard deviation of heart rate variability components analyzed in the time and frequency domains pre- and post- 16 weeks of training or sedentary control.

\begin{tabular}{|c|c|c|c|c|c|}
\hline \multirow{2}{*}{ iRR(ms) } & pre & $912.00 \pm 202.22$ & $865.25 \pm 75.79$ & $920 \pm 154.88$ & $887.38 \pm 125.88$ \\
\hline & post & $939.00 \pm 122.40$ & $866.75 \pm 140.98$ & $1003.75 \pm 128.20$ & $848.12 \pm 96.18$ \\
\hline $\mathrm{LF}\left(\mathrm{ms}^{2}\right)$ & pre & $194.81 \pm 261.99$ & $85.24 \pm 92.36$ & $295.88 \pm 489.16$ & $72.10 \pm 73.23$ \\
\hline \multirow{2}{*}{$\mathrm{LF}(\mathrm{nu})$} & pre & $58.12 \pm 20.34$ & $57.65 \pm 16.55$ & $63.68 \pm 15.01$ & $57.62 \pm 23.73$ \\
\hline & post & $52.67 \pm 18.67$ & $58.24 \pm 22.00$ & $61.21 \pm 18.73$ & $62.15 \pm 14.53$ \\
\hline $\mathrm{HF}\left(\mathrm{ms}^{2}\right)$ & pre & $91.09 \pm 107.79$ & $53.41 \pm 48.91$ & $104.15 \pm 160.25$ & $58.91 \pm 68.49$ \\
\hline $\mathrm{HF}(\mathrm{nu})$ & post & $47.32 \pm 18.67$ & $41.75 \pm 22.00$ & $38.78 \pm 18.73$ & $37.83 \pm 14.53$ \\
\hline \multirow{2}{*}{$\mathrm{LF} / \mathrm{HF}$} & pre & $1.93 \pm 1.33$ & $1.61 \pm 0.75$ & $2.37 \pm 1.92$ & $2.45 \pm 2.56$ \\
\hline & post & $1.49 \pm 1.20$ & $2.22 \pm 2.11$ & $2.24 \pm 1.84$ & $2.06 \pm 1.31$ \\
\hline \multirow{2}{*}{ RMSSD (ms) } & pre & $168.92 \pm 226.90$ & $24.23 \pm 10.05$ & $28.21 \pm 19.70$ & $29.44 \pm 11.56$ \\
\hline & post & $68.08 \pm 80.53$ & $24.55 \pm 13.91$ & $65.41 \pm 54.34$ & $38.92 \pm 53.29$ \\
\hline \multirow{2}{*}{$\mathrm{SD} 2(\mathrm{~ms})$} & pre & $151.47 \pm 167.02$ & $43.32 \pm 17.89$ & $58.36 \pm 42.63$ & $47.36 \pm 14.08$ \\
\hline & post & $73.59 \pm 49.00$ & $39.23 \pm 19.01$ & $89.50 \pm 44.22$ & $45.20 \pm 37.72$ \\
\hline
\end{tabular}

MSC = male sedentary control; $\mathrm{FSC}=$ female sedentary control; $\mathrm{MCT}=$ male concurrent training FCT = female concurrent training. 
The TABLE 4 demonstrates the evaluation of cardiorespiratory values and muscle strength variables before and after the training period for the groups. Muscle strength significant improvements was observed for the MCT in the leg press $(\mathrm{p}=$ $0.0001)$ and arm curl (0.0001) when compared to the sedentary groups. For FCT, a significant increase was observed only in the bench press ( $\mathrm{p}$ $=0.0130)$ and arm curl (0.0109) when compared to the sedentary groups. In the cardiorespiratory evaluation, significant differences were observed for the MCT ( $p=0.0001)$ and FCT $(p=0.0238)$. There was no significant difference in $\mathrm{VO} 2$ peak from the baseline for the sedentary groups.

TABLE 4- Mean \pm standard deviation of VO2peak and 1 repetition maximum test (bench press, leg press and arm curl) before and after 16 weeks of training protocols.

\begin{tabular}{|c|c|c|c|c|c|}
\hline & & MSC & FSC & MCT & FCT \\
\hline \multirow{2}{*}{$\begin{array}{l}\mathrm{VO}_{2 \text { peak }} \\
(\mathrm{ml} / \mathrm{kg} / \mathrm{min})\end{array}$} & pre & $32.23 \pm 4.43$ & $27.33 \pm 2.96$ & $31.63 \pm 5.37$ & $26.18 \pm 3.00$ \\
\hline & post & $32.14 \pm 3.08$ & $25.96 \pm 3.06$ & $35.78 \pm 5.23^{*}$ & $28.41 \pm 2.34^{*}$ \\
\hline \multirow{2}{*}{ Bench press $(\mathrm{k}$} & pre & $62.75 \pm 10.08$ & $39.25 \pm 6.15$ & $68.88 \pm 5.79$ & $39.87 \pm 4.58$ \\
\hline & post & $63.50 \pm 9.05$ & $42.50 \pm 6.23$ & $86.13 \pm 9.78^{*}$ & $46.75 \pm 6.62^{*}$ \\
\hline \multirow{2}{*}{ Leg press $(\mathrm{kg})$} & pre & $208.00 \pm 35.97$ & $133.37 \pm 34.25$ & $190.63 \pm 28.02$ & $151.87 \pm 23.21$ \\
\hline & post & $246.88 \pm 61.58$ & $158.12 \pm 18.31$ & $315.25 \pm 67.74^{*}$ & $172.12 \pm 38.87$ \\
\hline \multirow{2}{*}{ Arm curl $(\mathrm{kg})$} & pre & $35.50 \pm 3.20$ & $21.87 \pm 3.04$ & $36.75 \pm 3.06$ & $23.50 \pm 3.81$ \\
\hline & post & $37.63 \pm 4.40$ & $25.25 \pm 3.24$ & $46.50 \pm 4.69^{*}$ & $26.00 \pm 2.13^{*}$ \\
\hline
\end{tabular}

\section{Discussion}

The main findings of this study were that training protocol was not effective to alter resting HRV and $\mathrm{BP}$, even though the $\mathrm{VO}_{2 \text { peak }}$ improved for both training groups. In addition, both training groups have shown an increase in maximum upper body strength, but just men have increase in the lower limbs strength following concurrent training. Thus, these results contradict our hypothesis that 16 weeks of concurrent training would be able to promote similar gains in body strength and cardiac autonomic markers for both genders.

Some authors report that the association between aerobic and resistance training can lead to strength gains and cardiorespiratory fitness gains ${ }^{24}$, and improve the prevention of cardiovascular disease. However, there is little information regarding autonomic adaptations, particularly related to concurrent training ${ }^{24}$. Furthermore, the different training methods used hinder comparisons between the results ${ }^{39,40}$. In this study, with regard to the variables related to muscular strength indicators in response to the proposed training protocol, the assumption of gains and no 'interference effect' for untrained subjects when concurrent training is prescribe was negated ${ }^{41}$. Although, studies have shown strength gains similar to those from a specific training protocol for the development of muscle strength $^{42,43}$. However, few studies have investigated 
the effects of interference in the development of maximum strength in middle-aged individual $s^{40}$.

Regarding blood pressure levels and HR, a recently published meta-analysis ${ }^{8}$, the results showed that combined training do not reduce SBP and MAP and just significantly reduce DBP. In according, combined training proposed by us showed no reduction in any BP variable. One might imagine that the lack of effect of our training is due to the normal or little altered BP levels of our individuals. However, have been shown that in individuals with normal blood pressure or prehypertensive training also effects positively SBP and DBP, demonstrating the power of training as adjunctive therapy for high BP prevention in these populations ${ }^{8}$. In the present study a decrease in these BP values was not observed, although comparisons with literature are difficult due to the different loads, intensities and type of training used, other studies should comparer the effects of concurrent training in BP values of normal BP and hypertensive subjects.

Studies on concurrent training and HRV are scarce. Karavirta et al. ${ }^{12}$ showed improvements in cardiac dynamics in response to concurrent training (resting HR and HRV indicators) over 21 weeks of training in older men. In addition, significant changes were observed only for the concurrent training when compared to other training protocols used (aerobic and with weights). There was a negative correlation between the reduction in HR at rest and increased HF $(r=-0.81, p<0.001)$, which indicated greater vagal influence on cardiac autonomic function in the group undergoing concurrent training. Although in this study thus it could be speculated that the larger training volume was responsible for the best results obtained by the concurrent training group. In addition, another study ${ }^{24}$ from the same group showed that the same protocol did not confer benefits on HRV scores in women.

The study of VERHEYDEN et al. ${ }^{44}$ evaluated the effects of a concurrent exercise training program on cardiac autonomic control in 14 sedentary men with a mean age of $62 \pm 6.1$ years. The training consisted of approximately 75 minutes of aerobic activity at $65-80 \%$ of heart rate reserve plus two sets of 20-30 RM (repetition maximum) with a moderate load. The results of this study demonstrated no significant changes in HRV parameters at rest, and did not provide evidence of a significant increase in vagal modulation at rest after a year. These results suggest that the manipulation of these training variables may be of great importance for obtaining improvements in cardiac control in this population. However, more research is needed to clarify this information with regard to equalizing training volumes.

According to the Dutra et al. ${ }^{20}$ study, cardiac modulation differs between the genres, showing greater influence of the vagal autonomic component in women and the sympathetic component in men. Our results show no difference between the genres, neither in the baseline nor after the training protocol. Although a decline in earlier autonomic control in men may occur early because of the cardioprotective effect of estrogen ${ }^{19}$, in our study these differences were not found and one possible explanation is that women are postmenopausal. With regard to gender dependency, in the present study no differences in HRV variables were observed for any of the periods studied, corroborating data from another study ${ }^{21}$ which reported no differences in cardiovascular autonomic responses, HRV variables.

Despite considering only the improvements expected from specific programs of physical training for the variables related to the cardiovascular and respiratory systems, it is noteworthy that the evidence from the present study suggests that the systematic practice of physical exercise produces protective effects against the development of chronic degenerative diseases in advance stages of life, probable helping not only providing an increase in life expectancy, but above all improving the health status of an individual, making exercise a crucial public health strategy.

The changes induced by training in the modulation of HRV were not observed in the present study. However, Karavirta et al. ${ }^{24}$ study suggests that it may be possible to detect more subtle changes when measured during exercise, but not when measured at rest. This may have been a limitation of this study since the proposed training may be consideration of moderate-high intensity. Another limitation was the lack of homonal dosage in women, since at that age, it is suggested that women have lost considered levels of the hormone estrogen, which has cardioprotective effect in women.

Based on the results of the present study, it was concluded that the training protocol proved to be a good proposal for improving cardiorespiratory capacity and muscle strength particularly in mem, however without causing alterations in the analyzed cardiac autonomic indicators and BP. These finds suggest a possible interference 
effect' on cardiovascular variables, but we can not prove this assumption without comparison with the isolated aerobic training protocol. Further studies should compare the effects of aerobis and concurrent trainng protocols in middle-age men and women upon cardiovascular variables. In addition the manipulation of training volume may increase concurrent training effects, this hypothesis can also be test by other studies.

The changes induced by training in the modulation of HRV were not observed in the present study. However, Karavirta ${ }^{24}$ study suggests that it may be possible to detect more subtle changes when measured during exercise, but not when measured at rest. This may have been a limitation of this study since the proposed training may be consideration of moderate-high intensity. Another limitation was the lack of homonal dosage in women, since at that age, it is suggested that women have lost considered levels of the hormone estrogen, which has cardioprotective effect in women.

\section{Acknowledgments}

The authors wish to thank Financial support was provided by the National Counsel of Technological and Scientific Development, Brazil and the authors thank the volunteers for their enthusiastic commitment to this project.

\section{Disclosure of interest}

The authors declare that they have no conflicts of interest concerning this article.

\section{References}

1. Carlsson AC, Th eobald H, Wändell PE. Health factors and longevity in men and women: a 26-year follow-up study. Eur J Epidemiol. 2010;25:547-551.

2. Park MS, Chung SY, Chang Y, Kim K. Physical Activity and Physical Fitness as Predictors of All-Cause Mortality in Korean Men. J Korean Med Sci. 2009;24:13-9.

3. Booth FW, Roberts CK, Laye MJ. Lack of Exercise Is a Major Cause of Chronic Diseases. Compr Physiol. 2012;2:11431211.

4. Kraschnewski JL, Sciamanna CN, Poger JM, Rovniak LS, Lehman EB, Cooper AB, et al. Is strength training associated with mortality benefi ts? A 15 year cohort study of US older adults. Preventive Medicine. 2016;87:121-127.

5. Nelson ME, Rejeski WJ, Blair SN, Duncan PW, Judge JO, King AC, et al. Physical activity and public health in older adults: recommendation from the American College of Sports Medicine and the American Heart Association. Circulation. 2007;116:1094-105.

6. Chodzko-Zajko WJ, Proctor DN, Fiatarone Singh MA, Minson CT, Nigg CR, Salem GJ, et al. American College of Sports Medicine position stand. Exercise and physical activity for older adults. Med Sci Sports Exerc. 2009;41:1510-1530. 7. Garber CE, Blissmer B, Deschenes MR, Franklin BA, Lamonte MJ, Lee I, et al. American College of Sports Medicine position stand. Quantity and quality of exercise for developing and maintaining cardiorespiratory, musculoskeletal, and neuromotor fitness in apparently healthy adults: guidance for prescribing exercise. Med Sci Sports Exerc. 2011;43(7):1334-59. 8. Cornelissen VA, Smart NA. Exercise training for Blood Pressure: A Systematic Review and Meta-analysis. J Am Heart Assoc. 2013;27:1-9.

9. Montero D, Vinet A, Roberts CK. Eff ect of combined aerobic and resistance training versus aerobic training on arterial stiffness. Int. J. Cardiol. 2015;178:69-76.

10. TASK FORCE of European Society of Cardiology and North American Society of Pacing and Eletrophysiology. Heart Rate Variability. Circulation. 1996;93:1043-1065.

11. Malpas SC. Sympathetic Nervous System Overactivity and Its Role in the Development of Cardiovascular Disease. Physiol Rev. 2010;90:513-557. 
12. Karavirta L, Tulppo MP, Laaksonen DE, Nyman K, Laukkanen RT, Kinnunen H, et al. Heart rate dynamics after combined endurance and strength training in older men. Med Sci Sports Exerc. 2009;41:1436-43.

13. De Meersman RE, Stein PK. Vagal Modulation and Aging. Biol Psyc. 2008;74:165-73.

14. Stein PK, Barzilay JI, Chaves PHM, Mistretta SQ, Domitrovich PP, Gottdiener JS, et al. Novel measures of heart rate variability predict cardiovascular mortality in older adults independent of traditional cardiovascular risk factors: the Cardiovascular Health Study (CHS). J Cardiovasc Electr. 2008;19:1169-1174.

15. Buchheit M, Simon C, Viola AU, Doutreleau S, Piquard F, Brandenberger G. Heart rate variability in sportive elderly: relationship with daily physical activity. Med Sci Sport Exer. 2004;36:601-605.

16. Hansen AL, Johnsen BH, Sollers JJ, Stenvik K, Th ayer JF. Heart rate variability and its relation to prefrontal cognitive function: the eff ects of training and detraining. Eur J Appl Physiol. 2004;93:263-272.

17. Vanderlei LCM, Pastre CM, Hoshi RA, Carvalho TD, Godoy MF. Noções básicas de variabilidade da frequência cardíaca e sua aplicabilidade clínica. Rev Bras Cir Cardiovasc. 2009;24:205-217.

18. Soares-Miranda L, Sattelmair J, Chaves P, Duncan GE, Siscovick DS, Stein PK, et al. Physical activity and heart rate variability in older adults: the cardiovascular health study. Circulation. 2014;5:113.

19. Christodoulakos GE, Lambrinoudaki IV, Botsis DC. Th e cardiovascular eff ects of selective estrogen receptor modulators. Acad Sci. 2006;12:374-384.

20. Dutra SGV, Pereira APM, Tezini GCSV, Mazon JH, Martins-Pinge MC, et al. Cardiac autonomic modulation is determined by gender and is independent of aerobic physical capacity in healthy subjects. PLoS ONE. 2013;8(10):e77092. 21. Laitinen T, Niskanen L, Geelen G, Länsimies E, Hartikainen J. Age dependency of cardiovascular autonomic responses to head-up tilt in healthy subjects. J Appl Physiol. 2004;96:2333-2340.

22. Leite ST, Martinelli FS, Madruga VA, Catai AM, Gallo Junior L, Chacon-Mikahil MPT. Respostas cardiovasculares a mudança postural e capacidade aeróbia em homens e mulheres de meia-idade antes e após treinamento físico aeróbio. Rev Bras Fisiot. 2009;12:392-400.

23. Sloan RP, Shapiro PA, DeMeersman RE, Bagiella E, Brondolo EN, McKinley PS, et al. The effect of aerobic training and cardiac autonomic regulation in young adults. Am J Public Health. 2009;99(5):921-8.

24. Karavirta L, Costa MD, Goldberger AL, Tulppo MP, Laaksonen DE, Nyman K, et al. Heart Rate Dynamics after combined strength and endurance training in middle-aged women: heterogeneity of responses. PLoS ONE. 2013;8(8):e72664.

25. Dantas APV. Açóes cardiovasculares dos hormônios sexuais femininos. Hipertensão. 2005;8:86-90.

26. Liu CC, Terry BJK, Cheryl CHY. Eff ects of estrogen on gender-related autonomic diff erences in humans. Am J Physiol Heart Circ Physiol. 2003;285:H2188-H2193.

27. Okada Y, Galbreath MM, Shibata S, Jarvis SS, VanGundy TB, Meier RL, et al. Relationship between sympathetic barorefl ex sensitivity and arterial stiff ness in elderly men and women. Hypertension. 2012; 59:98-104.

28. Voorrips LE, Ravelli AC, Petra C, Deurenberg P, Staverenet WA. A physical activity questionnaire for the elderly. Med Sci Sports Exerc. 1991;23:974-9.

29. Pardini R, Matsudo S, Araújo T, Matsudo V, Andrade E, Braggion G, et al. Validation of the International Physical Activity Questionaire (IPAQ version 6): pilot study in Brazilian young adults. Rev Bras Ciên Mov. 2001;9:45-51.

30. Gordon CC, Chumlea WC, Roche AF. Stature, Recumbent Length, Weight. In: Lohman, TG, ed. Anthropometric Standardizing Reference Manual. Champaign, Illinois: Human Kinetics Books; 1988:3-8.

31. Gamelin FX, Berthoin S, Bosquet L. Validity of the polar S810 heart rate monitor to measure R-R intervals at rest. Med Sci Sports Exerc. 2006;38:887-93.

32. Nunan D, Donovan G, Jakovljevic D, Hodges LD, Sandercock GRH, Brodie DA. Validity and reliability of shortterm heart-rate variability from the Polar S810. Med Sci Sports Exerc. 2009;41:243-50.

33. Malik M. Heart Rate Variability: Standards of Measurement, Physiological Interpretation, and Clinical Use. Task Force of European Society of Cardiology and the North American Society of Pacing and Eletrophysiology. Circulation. 1996;93:1043- 1065.

34. Libardi CA, Souza GV, Gaspari AF, et al. Eff ects of concurrent training on interleukin-6, tumour necrosis factor-alpha and C-reactive protein in middle-aged men. J Sports Sci 2011; 29 (14):1573-1581.

35. Wasserman K, Whipp BJ, Koyl SN, Beaver WL. Anaerobic threshold and respiratory gas exchange during exercise. J Appl Physiol. 1973;35:236-243.

36. Brown LE, Joseph P, Weir JP. (ASEP) Procedures Recommendation I: Accurate assessment of muscular strength and power. J Exerc Physiol online. 2001;4:1- 21.

37. ACSM. Manual de Pesquisa das Diretrizes do ACSM para os Testes de Esforço e sua Prescrição. 4. ed. Rio de Janeiro: 
Guanabara Koogan, 2002.

38. ACSM Position Stand. The recommended quantity and quality of exercise for developing and maintaining cardiorespiratory and muscular fitness, and flexibility in healthy adults. Med Sci Sports Exerc. 1998;30:975-991.

39. Häkkinen K, Alen M, Kraemer WJ, Gorostiaga E, Izquierdo M, Rusko H, et al. Neuromuscular adaptations during concurrent strength and endurance training versus strength training. Eur J Appl Physiol. 2003;89:42-52.

40. Izquierdo M, Häkkinen K, Ibáńez J, Kraemer WJ, Gorostiaga EM. Eff ects of combined resistance and cardiovascular training on strength, power, muscle cross-sectional area, and endurance markers in middle-aged men. Eur J Appl Physiol. 2005;94:70-5.

41. Coff ey VG, Hawley JA. Concurrent exercise training: do opposites distract? J Physiol. 2017;595.9:2883-2896.

42. Docherty D, Sporer B. A proposed model for examining the interference phenomenon between concurrent aerobic and strength training. Sports Med. 2002;30:385-94.

43. Cadore EL, Pinto RS, Lhullier FLR, Correa CS, Alberton CL, Pinto SS, et al. Physiological eff ects of concurrent training in elderly men. Int J Sports Med. 2010;31:689-97.

44. Verheyden B, Eijnde BO, Beckers F. Low-dose exercise training does not infl uence cardiac autonomic control in healthy sedentary men aged 55 - 75 years. J Sport Sci. 2006;24:1137-1147.

ADDRESS

Melissa Antunes

Otávio Cesário Pereira street, 138 - Acapulco 86045-020 - Londrina - PR - Brazil E-mail: melissa.antunes@hotmail.com
Submitted: 02/07/2017

Revised: 08/31/2017

Accepted: 08/22/2018 